IOS Press

\title{
FGF1 containing biodegradable device with peripheral nerve grafts induces corticospinal tract regeneration and motor evoked potentials after spinal cord resection
}

\author{
Jonathan Nordblom ${ }^{\mathrm{a}, \mathrm{b}}$, Jonas K.E. Persson ${ }^{\mathrm{a}, \mathrm{d}}$, Jonas Åberg ${ }^{\mathrm{e}}$, Hans Blom ${ }^{\mathrm{f}}$, Håkan Engqvist ${ }^{\mathrm{e}}$, \\ Hjalmar Brismar ${ }^{\mathrm{f}}$, Johan Sjödahl ${ }^{\mathrm{g}}$, Anna Josephson ${ }^{\mathrm{h}}$, Arvid Frostell ${ }^{\mathrm{a}}$, Sebastian Thams ${ }^{\mathrm{a}}$, \\ Lou Brundin $^{\mathrm{a}, \mathrm{c}}$, Mikael Svensson ${ }^{\mathrm{a}, \mathrm{b}, *}$ and Per Mattsson ${ }^{\mathrm{a}}$ \\ ${ }^{a}$ Department of Clinical Neuroscience, Karolinska Institute, Stockholm, Sweden \\ ${ }^{\mathrm{b}}$ Department of Neurosurgery, Karolinska University Hospital, Stockholm, Sweden \\ ${ }^{\mathrm{c}}$ Department of Neurology, Karolinska University Hospital, Stockholm, Sweden \\ ${ }^{\mathrm{d}}$ Department of Neurophysiology, Karolinska University Hospital, Stockholm, Sweden \\ ${ }^{\mathrm{e}}$ Materials in Medicine, Uppsala University, Uppsala, Sweden \\ ${ }^{\mathrm{f}}$ Cell Physics, Royal Institute of Technology, Stockholm, Sweden \\ ${ }^{\mathrm{g}}$ BioArctic Neuroscience AB, Stockholm, Sweden \\ ${ }^{\mathrm{h}}$ Department of Neuroscience, Karolinska Institute, Stockholm, Sweden
}

\begin{abstract}
Purpose: Repairing the spinal cord with peripheral nerve grafts (PNG) and adjuvant acidic fibroblast growth factor (FGF1) has previously resulted in partial functional recovery. To aid microsurgical placement of PNGs, a graft holder device was previously developed by our group. In hope for a translational development we now investigate a new biodegradable graft holder device containing PNGs with or without FGF1.

Methods: Rats were subjected to a T11 spinal cord resection with subsequent repair using twelve white-to-grey matter oriented PNGs prepositioned in a biodegradable device with or without slow release of FGF1. Animals were evaluated with BBB-score, electrophysiology and immunohistochemistry including anterograde BDA tracing.

Results: Motor evoked potentials (MEP) in the lower limb reappeared at 20 weeks after grafting. MEP responses were further improved in the group treated with adjuvant FGF1. Reappearance of MEPs was paralleled by NF-positive fibers and anterogradely traced corticospinal fibers distal to the injury. BBB-scores improved in repaired animals.

Conclusions: The results continue to support that the combination of PNGs and FGF1 may be a regeneration strategy to reinnervate the caudal spinal cord. The new device induced robust MEPs augmented by FGF1, and may be considered for translational research.
\end{abstract}

Keywords: Spinal cord injuries, motor evoked potential, rats, peripheral nerves, fibroblast growth factor 1, tracing, neurophysiology, axonal regeneration

\footnotetext{
${ }^{*}$ Corresponding author: Mikael Svensson, MD, PhD, Department of Clinical Neuroscience, Karolinska Institute and Department of Neurosurgery, Karolinska University Hospital, SE-171 76 Stockholm, Sweden. Tel.: +46 85177 0000; Fax: +46 85177 1778; E-mail: mikael.a.svensson@karolinska.se.
} 


\section{Introduction}

Spinal cord injury (SCI) remains a major challenge in neuroscience. A number of promising strategies to repair CNS injuries have been presented in recent years and several clinical trials have been or are about to be launched in the near future (Fehlings et al., 2011; Kwon et al., 2010; Wu et al., 2011). Many strategies are focused on optimizing the conditions after acute or subacute spinal cord injury. The aims are mainly to limit secondary reactions, prevention of delayed axonal/neuronal damage, regeneration across the injury, improvement of the local milieu etc (Bunge, 2008; Cadotte and Fehlings, 2010; Schwab et al., 2006). Recently, new hope was raised for chronic SCI patients when epidural stimulation of the caudal spinal cord in combination with locomotor training resulted in voluntary movements of the lower extremities (Harkema et al., 2011). The method is thought to utilize previously clinically silent axons crossing the lesion. Potentially, even a complete and chronic SCI with no spared axons may result in voluntary movements of lower extremities if a regeneration strategy is applied in combination with locomotor training and possibly epidural stimulation.

The use of peripheral nerve grafts (PNG) could be a possible strategy to partly regain functions of the injured spinal cord (Alilain et al., 2011; Cote et al., 2011). Some experiments use PNGs in combination with acidic fibroblast growth factor (FGF1) and practise the strategy of rerouting regenerating fibers in the white matter into adjacent grey matter (Cheng et al., 1996; Lee et al., 2008; Lee et al., 2002; Lee et al., 2004; Tsai et al., 2005). The rerouting strategy is intended to avoid white matter growth inhibitory compounds such as NOGO and other myelin associated proteins or repelling extracellular matrix components (Chen et al., 2000; Fitch and Silver, 2008; Schwab, 2004). Experiments presented by Tsai and co-workers favoring this hypothesis showed that longitudinal tracts directed to white or grey matter on the opposite side of a transection gap in the spinal cord prefer growing into grey matter regardless of guidance (Tsai, et al., 2005).

Meticulous dissection of the PNGs and the adjacent CNS tissue aiming at distinct positioning of PNGs seems to be mandatory in achieving axonal growth. We have in a recent study shown that the precision of the surgical procedure can be improved and easily reproduced using a holder for the PNGs (called device below) (Nordblom et al., 2009). Insertion of a spinal cord device would require a spinal cord resection in the patient, which theoretically could be performed as a clinical surgical intervention in chronic and complete SCI, provided that no long tract axons are in continuity across the lesion.

Considering a possible translational development, where a device with PNGs is used in combination with FGF1, the patient would likely benefit from a biodegradable device to avoid long-term effects of implanted material in the CNS. Delivery of FGF1 could be carried out through integration in the device. Therefore, we have developed a device made of calcium sulphate, which meets the requirements of biodegradability and slow release of FGF1 (Aberg et al., 2010). The biological effect of this new device with PNGs is unknown, with or without FGF1.

The aim of the present study was to investigate if i) the new device containing autologous PNGs could result in a motor cortex derived electrophysiological response in the hind limb (MEP), ii) if the addition of FGF1 would improve the MEP response, and iii) motor cortex derived hind limb response was paralleled by motor cortex neuron regeneration across the injury zone.

\section{Materials and methods}

\subsection{The biodegradable graft device}

The principles concerning the construction of the graft device using dental cement was recently documented in detail (Nordblom, et al., 2009). In the present study we use the same procedure, however changing the material from dental cement to calcium sulphate, which is biodegradable. The rationale for using a biodegradable material was based on the idea that non-dissolving artificial material presented to CNS tissue in the long perspective may trigger local immune responses with cyst formations and furthermore act as a substrate for potential bacterial infections that may appear years after surgery.

The $\alpha$-calcium sulphate hemihydrate powder (Bo Ehrlander AB, Gothenburg, Sweden) was mixed with deionized water (liquid/powder ratio 0.35 ) to a homogenous paste and injected into a small cistern containing plastic wires representing the projections of the later inserted peripheral nerve grafts (PNG). The cistern was vibrated for 90 seconds and left to harden in room temperature for 24 hours. 
$\mathrm{a}$
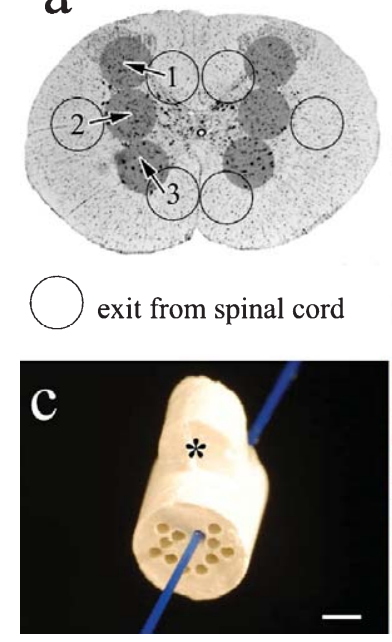

b
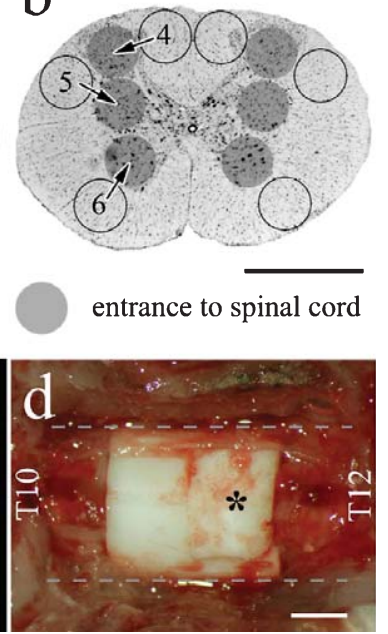

Fig. 1. a. Schematic view indicating six channels receiving descending motor tracts from white matter (empty circles), projecting them toward caudal grey matter in the distal spinal cord (grey filled circles represent landing positions in the resection gap $3 \mathrm{~mm}$ caudally). 1: Dorsal Corticospinal, 2: Lateral Corticospinal, 3: Ventral Corticospinal. b: Additional six channels projecting ascending sensory tracts from the caudal white matter (empty circles) to grey matter cranially (grey circles represent landing positions in the resection gap $3 \mathrm{~mm}$ cranially). 4: Cunate/Gracile, 5: Spinocerebellar, 6: Spinothalamic. Bar $=1 \mathrm{~mm}$. c: Hardened calcium sulphate device, cranial view. The 12 visible channels oriented according to Fig. 1a and b, representing entrances of six descending motor tracts and exits of six ascending sensory tracts (see text above). A blue Ethilone ${ }^{\circledR}$ suture indicates the left dorsal corticospinal tract. For orientation, the device had a dorsal indicator $(*)$ that was removed with a small bone rongeur after placement in the spinal cord gap. Bar $=1 \mathrm{~mm}$. d: photograph of implanted device between spinal cord stumps at T11. The dorsal indicator has been removed $(*)$. Bar $=1 \mathrm{~mm}$.

The wires were removed after hardening of the material, creating channels where peripheral nerve grafts could be inserted. Six channels were positioned representing descending motor tracts in the white matter projecting to adjacent grey matter caudally and another six channels represented ascending sensory tracts projecting to adjacent grey matter cranially (Fig.1a and b), in accordance with Nordblom and colleagues (Nordblom, et al., 2009). However in this study we focused only on regeneration in the descending corticospinal fibers. The size of the device measured $3.0 \mathrm{~mm}$ in height, $3.0 \mathrm{~mm}$ in diameter and contained 12 channels with a diameter of $0.38 \mathrm{~mm}$ each (Fig. 1c). The nanoporous structure of the calcium sulphate permitted loading and a controlled slow release of FGF1 (Aberg, et al., 2010).

\subsection{Acidic fibroblast growth factorl (FGF1)}

For growth factor delivery, the device described above was incubated to absorb FGF1 in concentrations of $0.05 \mathrm{mg} / \mathrm{ml}$ or $0.5 \mathrm{mg} / \mathrm{ml}$ (Protein Sciences, Meriden, CT, US) during three days at $+4^{\circ} \mathrm{C}$ in a phosphate buffered saline solution (PBS, Sigma Aldrich, St Louis, MO, US), with heparin (concentration $1: 1 \mathrm{w} / \mathrm{w}$, heparin sodium salt from porcine intestinal mucosa, Sigma Aldrich, St Louis, MO, US). Adding heparin to FGF1 has previously been shown to stabilize and enhance the activity of this growth factor (Aberg, et al., 2010). The graft device absorbed approximately $10 \mu \mathrm{l}$ of FGF1/heparin fluid, corresponding to a total dose of $0.5 \mu \mathrm{g}$ or $5 \mu \mathrm{g}$, respectively. Prepared graft devices were stored in $-20^{\circ} \mathrm{C}$ before surgery.

\subsection{Surgery}

Adult (270-290g) female Sprague Dawley rats (Scanbur $^{\circledR}$, Sollentuna, Sweden) were used in all experiments. All animals $(n=48)$ were kept in ventilated, humidity- and temperature controlled rooms with 12 hour light per 24-hour cycle and received food pellets and water ad libitum, according to regulations at Karolinska Institutet. All experiments were approved by the Stockholm Animal Ethics Committee. Surgery was carried out as follows:

1) Spinal cord transection and resection (SCI) of $3 \mathrm{~mm}$ of the cord at T11 followed by positioning of grafts using the device described above without FGF1 $(n=8)$, or

2) SCI and grafting using a device with a low dose of absorbed FGF1 $(0.5 \mu \mathrm{g}, n=8)$ or a high dose FGF1 $(5 \mu \mathrm{g}, n=8)$.

3) A third group was designed to specifically trace corticospinal axons by injection of BDA in the right motor cortex area. These animals were subjected to SCI and grafting as described above (see group 2, $n=6$ ), or SCI without grafts or FGF1 $(n=6)$.

Two additional control groups included sham operated animals subjected to laminectomy leaving the spinal cord intact $(n=4)$ or animals subjected to a SCI as described above, however without grafting and growth factors $(n=8)$.

The animals were anaesthetized with continuous isoflurane inhalation (2.2-2.7\%), and kept warm with a thermostatic heating pad. Heart rate and oxygen 
saturation was measured in the paw, and the rats were given extra oxygen together with isoflurane to keep saturation above $95 \%$.

A Leica operating microscope was used during the whole procedure. After a dorsal midline skin incision, a laminectomy of the T11 vertebra was performed with high-speed diamond drills (1.0 and $0.5 \mathrm{~mm}$ in diameters, Anspach E-Max 2, Palm Beach Gardens, FL, US). The rats were subjected to a $3 \mathrm{~mm}$ resection of the spinal cord at vertebra level T11 (neurological level T12), performed with micro scissors in high magnification. For repair, twelve branches from autologous intercostal nerves were harvested and positioned in the channels of the graft device. The nerve stumps were trimmed in high magnification with micro scissors at the entrances and exits of the channels.

The device was positioned in the spinal cord gap (Fig. 1d) and an indicator in the midline of the device provided dorso-ventral and cranio-caudal orientation (Fig. 1c). After adequate placement, the dorsal indicator at the back of the device was gently removed with a small bone rongeur (Fig. 1d). Muscle and skin were closed in layers. Antibiotics were administered in the drinking water postoperatively (Sulfadoxin $1.14 \mathrm{mg} / \mathrm{ml}$ and Trimetoprime $0.23 \mathrm{mg} / \mathrm{ml}$; BorgalVet ${ }^{\circledR}$, Intervet International B.V.). Postoperative analgesia was given subcutaneously for three days (Caprofen $5 \mathrm{mg} / \mathrm{kg} \mathrm{BW} \times 2$ first day, then $\times 1$; Rimadyl ${ }^{\circledR}$ Vet, Pfizer and Buprenorphine $0.05 \mathrm{mg} / \mathrm{BW} \times 2$; Temgesic ${ }^{\circledR}$, Schering-Plough). Urinary bladders were emptied manually twice daily.

\subsection{Functional hind limb tests}

Hind limb function was evaluated in all animals by two observers once weekly using the Basso, Beattie and Bresnahan score (BBB) (Basso et al., 1995).

\subsection{Electrophysiology}

Electrophysiological analysis was performed 20 weeks post surgery using motor evoked potentials (MEPs). Stimulation thresholds were documented (gradual increase of the current until MEPs were registered), as well as latency time (time from stimulation to response). If no MEPs could be triggered, stimulation was interrupted at $30 \mathrm{~mA}$. MEPs from forelimbs as well as hind limbs were registered in all investigated animals.
After intraperitoneal ketamine/medetomidine anaesthesia $\quad\left(\right.$ Ketalar $^{\circledR}, \quad 75 \mathrm{mg} / \mathrm{kg}+$ Dormitor $^{\circledR}$, $0.5 \mathrm{mg} / \mathrm{kg}$ ) the rats were placed in a frame with ear pin holders. A midline skin incision was made to expose the skull bone, which was then drilled to expose the epidural surface of the motor cortex on each side. Medtronic Key Point equipment was used for the electrophysiology (Software version 5.03, Minneapolis, MN, US). The motor cortex was identified using anatomical landmarks and stimulated via a bipolar stimulator probe (Neurosign ${ }^{\circledR}$ Magstim, UK) placed epidurally on either hemisphere. Recording needles were inserted bilaterally into hamstring muscles and both forearm muscles, with the reference needles inserted into the respective muscle tendon about $1-2 \mathrm{~cm}$ distal to the active electrodes. The response in all four extremities were simultaneously recorded after stimulation of the right as well as left motor cortex. Stimulation was given with four pulses, stimulation duration $0.2 \mathrm{~ms}$, interstimulus interval $2 \mathrm{~ms}$, and stimuli were gradually elevated until reproducible responses were recorded. Stimulations were stopped at $30 \mathrm{~mA}$. A subcutaneous needle electrode in the tail root was used for grounding and a band pass filter set to $10-2000 \mathrm{~Hz}$ was used.

Every animal that showed positive MEPs in one or both hind limbs was re-lesioned with a complete SCI above level T8. New MEP-recordings were then undertaken to confirm that the previously recorded potentials had been caused by electrical conduction initiated and generated above the T8-level, to exclude that the current had traveled outside the spinal cord to the lower limbs. MEP-recordings from the upper extremities were registered to ensure that stimulation was given correctly also after the re-lesion.

\subsection{Anterograde tracing}

Seven weeks after surgery animals subjected to tracing (group 3, $n=6+6$ ) were re-anaesthetized and a midline skin incision was made on the skull. A minor part of the parietal bone was drilled away to expose the right motor cortex. An air pressure controlled micro glass pipette connected to a stereotactic frame was used and, in accordance with the previous electrophysiological identification of the correct neuron pools by positive MEPs to the hind limbs, four shallow injections (approximately $1.2 \mathrm{~mm}$ deep) of $0.5 \mu \mathrm{L} 10 \%$ biotinylated dextran amine (BDA; MW 10000; Molecular Probes, Leiden, The Netherlands) were made into 
the motor cortex, thus labeling motor cortex neurons projecting to the hind limbs. Three weeks later (10 weeks post grafting) these animals were sacrificed for histology.

\subsection{Tissue processing}

Immediately following the electrophysiology, animals were perfused through intracardiac infusion with body warm isotonic saline $\left(37^{\circ} \mathrm{C}\right)$ and thereafter cold paraformaldehyde $(4 \% \mathrm{w} / \mathrm{v})$. The spinal cords including the lesion areas were removed by meticulous dissection. In half of the rats a $12 \mathrm{~mm}$ segment of the spinal cord, with the injury zone placed in the middle, was cut transversely in $1 \mathrm{~mm}$ slices in a tissue matrice (Braintree Scientific, MA, US). Of the transverse slices, every second was put in $1.5 \%$ glutaraldehyde/4\% paraformaldehyde for semithin sectioning $(0.5-1 \mu \mathrm{m})$. Every other transverse slice was postfixed in paraformaldehyde $(4 \% \mathrm{w} / \mathrm{v})$ for two hours, rinsed in phosphate buffer with $17 \%$ (w/v) sucrose overnight for subsequent cryostat sections ( $14 \mu \mathrm{m})$, and mounted on slides. In the other half of the animals the spinal cords were postfixed in paraformaldehyde $(4 \% \mathrm{w} / \mathrm{v})$ for two hours, rinsed in phosphate buffer with $17 \%$ (w/v) sucrose overnight and subsequently $60 \mu \mathrm{m}$ longitudinal free floating cryostat sections were made.

\subsection{MRI studies}

Five animals were used for magnetic resonance imaging at one day or 10 weeks postoperatively. After intraperitoneal ketamine/medetomidine anaesthesia (Ketalar ${ }^{\circledR}, 75 \mathrm{mg} / \mathrm{kg}+$ Dormitor $^{\circledR}, 0.5 \mathrm{mg} / \mathrm{kg}$ ) the rats were subjected to MRI scannings. MRI recordings were performed using a Bruker BioSpec Avance $47 / 40$ spectrometer with a $4.7 \mathrm{~T}$ horizontal magnet with a $40 \mathrm{~cm}$ bore, used with a flat surface coil of $20 \mathrm{~mm}$ diameter. Images were acquired employing rare sequences (Hennig et al., 1986) with a field of view of $30 \times 30 \mathrm{~mm}$ with an effective echo time of $48.62 \mathrm{~ms}$ and a TR of $2.5 \mathrm{~s}$. A rare-factor of 8 was used with a rare maximum of 4 . The slice thickness was $1 \mathrm{~mm}$ and 192 in phase code and 256 in read out. NEX: 4

\subsection{Immunohistochemistry}

All cryostat sections were incubated in PBS with $1 \%$ bovine serum albumin (BSA, Sigma, St. Louis, USA), 0.3\% Triton-X-100 (Sigma, St. Louis,
USA) and $0.1 \%$ sodium azide in phosphate buffer $(0,15 \mathrm{M}, \mathrm{pH} 7.4)$ for 1 hour at room temperature. The transverse sections $(14 \mu \mathrm{m})$ were then incubated with antibodies against neurofilament protein (NF, Monoclonal Mouse, Anti-Human, Dako, Glostrup, Denmark, $1: 200$ ) or anti-glial fibrillary acidic protein (GFAP, Polyclonal Rabbit, Dako, Glostrup, Denmark, $1: 1000)$ at $4{ }^{\circ} \mathrm{C}$ overnight in PBS, and further incubated with secondary antibodies in room temperature for one hour (anti-mouse IgG conjugated to Alexa 488, A-21379, Invitrogen, Molecular Probes, Eugen, Oregon, USA, 1:100). Sections were mounted in Mowiol $^{\circledR}$ (Calbiochem, CA, US).

The free flowing longitudinal sections $(60 \mu \mathrm{m})$ were incubated with anti-NF $(1: 200)$ at $4^{\circ} \mathrm{C}$ in PBS for three days. The sections were then incubated with secondary antibodies (Alexa 488, see above, 1:500) over night at room temperature, rinsed and mounted on glass in Mowiol for analysis in the confocal microscope (Leica Microsystem TCS SP2 and Carl Zeiss LSM 5 Exciter).

In animals subjected to cortical injection of the tracer $\mathrm{BDA}$, the $60 \mu \mathrm{m}$ longitudinal free-floating sections were incubated with avidine conjugated to Alexa 488 (Invitrogen, Molecular Probes, Eugen, Oregon, USA, $1: 100$ ) for four hours at room temperature. Omission of primary or secondary antibodies was used as negative controls.

\subsection{Semithin sections}

The $1 \mathrm{~mm}$ transverse slices of the spinal cord of the lesion area were rinsed in phosphate buffer $(20 \mathrm{~min} \times 3)$ and immersed in osmium tetroxide (1\%) for two hours, rinsed in phosphate buffer, dehydrated in a graded series of ethanol to acetone, and embedded in agar resin 100. Semithin sections $(0.5 \mu \mathrm{m})$ were cut on an ultrotome, stained with toluidine blue dye (Chroma-Gesellschaft mbh \& Co. d-48161 Munster, Germany) and mounted with Pertex ${ }^{\circledR}$ (Histolab products, Gothenburg, Sweden) and cover glass. The morphological characteristics of the tissue were analyzed with light microscopy (Leica, $\times 100$ magnification, oil immersion).

\section{Results}

\subsection{Macroscopic post-mortem findings}

Graft devices were found degraded in all animals at both 10 and 20 weeks post surgery (Fig. 2a). All 

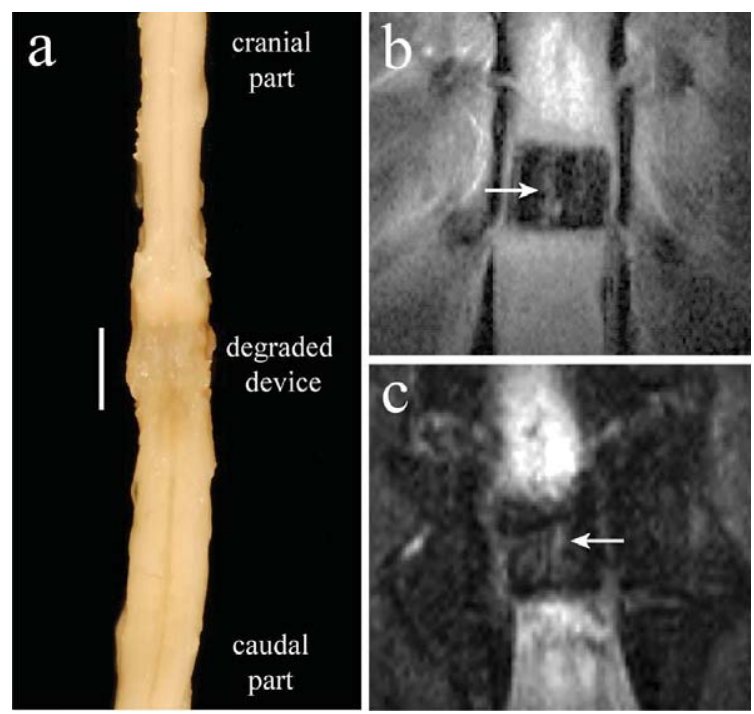

Fig. 2. a. Macroscopic post mortem finding of the perfused spinal cord 10 weeks after spinal cord repair with device and peripheral nerve grafts, anterior view, showing well integrated and degraded spinal cord device at level T11. Bar $=3 \mathrm{~mm}$. $2 \mathrm{~b}$ and $\mathrm{c}$ : MRI of the spinal cord repair area in the living animal at one day (b) and 10 weeks postoperatively (c). The MRI scans show the location of the device in the spinal cord. The implanted (b) and degraded (c) device (low MRI signal) is seen in the middle of the pictures, filling the resection gap. The device/degraded device seemed to be in placed position at one day as well as 10 weeks postop. Note the longitudinal structures with similar MRI signal as the spinal cord, most likely representing nerve grafts within the device (arrows).

individual grafts were fixed to the spinal cord surfaces on both sides at expected projection spots. There were no signs of infection, spine deformities or other unexpected macroscopic findings (Fig. 2a).

\subsection{MRI scanning}

One animal was investigated with MRI of the spinal cord at one day postoperatively and four rats were investigated 10 weeks postop. The implanted device seemed to be well in place the first post-operative day, projecting an MRI signal describing high water content. At ten weeks postop the device was found to be completely dissolved at dissection. The MRI at 10 weeks postop showed grafts running in the gap between in the divided cord. Furthermore, the MRI did not reveal any signs of cyst formations in the cord or other unexpected pathological findings such as infection or spine deformities (Fig. 2b, c).

\subsection{Functional scoring}

All animals scored 0 on the BBB scale the first postoperative weeks (Fig. 3), except from sham animals. From postoperative week 6-20, the repaired animals presented with scores between 0 and 4 , with significantly higher scores in repair groups in comparison to controls $(p=0.0001$, One Way ANOVA, $\mathrm{df}=3$, Bonferroni's multiple comparison test). Controls without graft and FGF1 scored 0 the first three weeks and throughout the remaining test period 0-1. Sham operated animals scored 21 points (normal hind limb function), at all BBB sessions (not shown).

\subsection{Electrophysiology}

Motor evoked potentials (MEPs) from the forelimbs were found in all animals with a preserved lateralization, i.e. unilateral motor cortex stimulation resulted in a contralateral response in the leg. MEPs from the hind limbs also presented with a clear lateralization, in some animals only in one leg (unilateral response) and in other animals in both legs (bilateral response). Representative MEP recordings are shown in Fig. 4.

In animals repaired with nerve grafts but with no adjuvant FGF1, five out of eight animals presented MEPs in hamstrings bilaterally (Fig. 5) after grafting, i.e. significantly improved in comparison to control animals subjected to SCI without grafting ( $p=0.031$, Fisher's exact test, $\mathrm{df}=1)$. The mean stimulatory threshold was $12.2 \pm 1.1 \mathrm{~mA}$ and mean latency time was $15.6 \pm 2.6 \mathrm{~ms}$. Two animals presented with a unilateral response and one animal failed to trigger potentials at all in the lower extremities.

All animals subjected to grafting and FGF1 treatment presented MEPs in hamstrings bilaterally (Fig. 5). The electrophysiological responses in this group were significantly improved compared to controls subjected to SCI without grafting ( $p=0.001$, Fisher's exact test, $\mathrm{df}=1$ ), but also better than the group treated with grafting without FGF1 ( $p=0.028$, Fisher's exact test, $\mathrm{df}=1$ ). Stimulatory thresholds with low dose adjuvant FGF1 and high dose FGF1 were $14.3 \pm 0.75 \mathrm{~mA}$ and $12.3 \pm 0.6 \mathrm{~mA}$ respectively and the mean latency time $12.3 \pm 1.7 \mathrm{~ms}$ and $18.5 \pm 2.5 \mathrm{~ms}$.

Reproducible MEPs were recorded bilaterally in all four sham cases $(n=4)$ with stimulation thresholds of $5.0 \pm 0.2 \mathrm{~mA}$ (mean \pm SEM) and latency time of $11.6 \pm 1.1 \mathrm{~ms}$ (mean \pm SEM) (Fig. 4), which was not significantly different from the repair groups. 


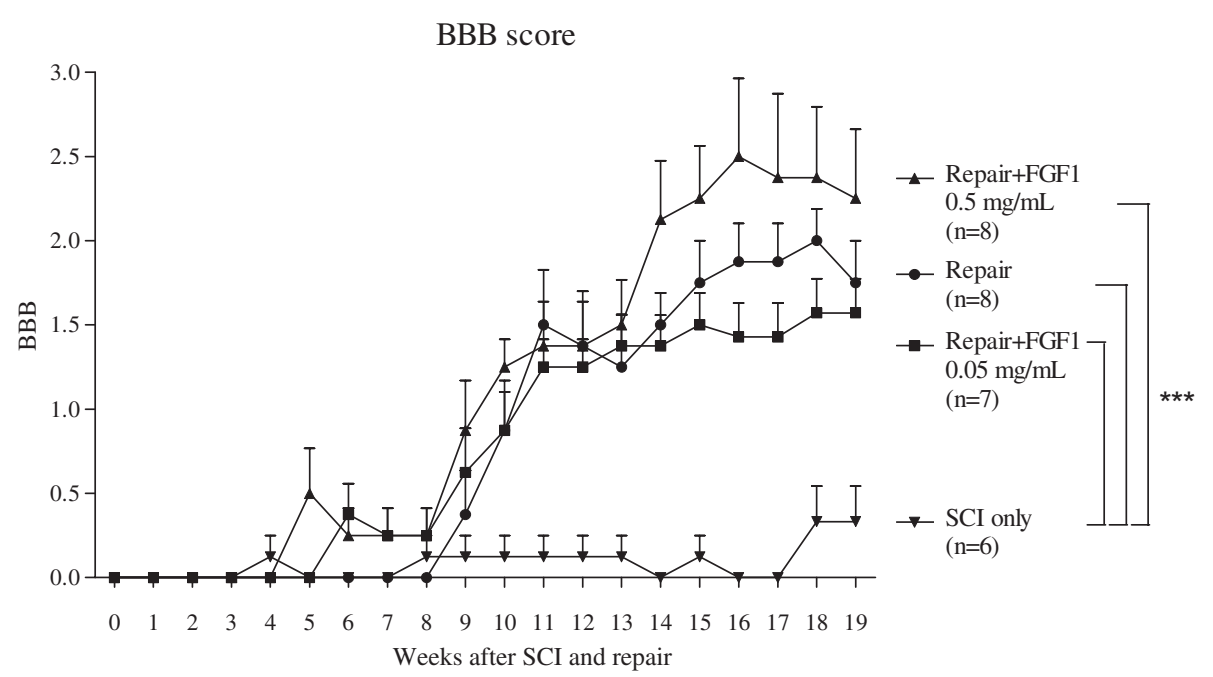

Fig. 3. The BBB scale showing functional recovery over the postoperative period (mean \pm SEM). The first four to six weeks, no or very little recovery was seen. The following weeks a modest, however, significant improvement could be seen in all repair groups, which was not seen in SCI controls ( $p=0.0001$, One Way ANOVA with Bonferroni's multiple comparison test). No significant difference was noted among the different repair groups.

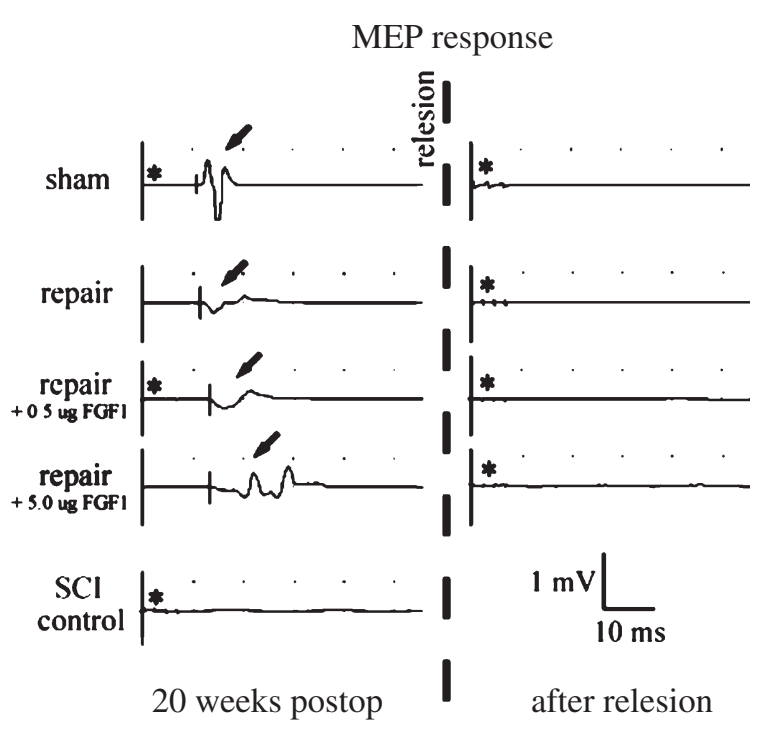

Fig. 4. Representative oscilloscope curves from animals subjected to sham operations, three different repair groups after SCI and SCI controls. Left column shows clear MEP responses (arrows) at 20 weeks postop except for SCI controls where no MEPs were detected. Right column shows absence of MEPs after re-lesion at the level of T10. Visible stimulation artifacts in some traces are indicated (asterisks).

Bilateral MEP responses could not be induced in any of the 6 investigated animals in the control group (SCI without grafting, Fig. 5). However, 2 of these animals showed a MEP response in one leg (stimulatory threshold $13.0 \pm 3.0 \mathrm{~mA}$, latency time $11.1 \pm 0.75 \mathrm{~ms}$ ).

Animals that presented MEPs were re-lesioned three segments above the repair zone, which in every case resulted in a complete loss of MEP responses in the hind limbs (Fig. 4), but preserved MEPs in both arms.

\subsection{Anterograde tracing}

To investigate the possibility of corticospinally derived regeneration through the spinal cord device, six animals were examined for presence of BDA in the spinal cord three weeks after injection into the motor cortex. Animals without grafting $(n=6)$ were used as controls. In both groups, numerous pyramidal cells presented BDA-IR at the injection site as well as thin, labeled fibers further down in the brain (Fig. 6g). Furthermore, we found columns with BDA positive fibers in the spinal cord, contralateral to the injection side, above the lesion area, representing uninjured parts of the corticospinal tract (Fig. 6h).

Numerous BDA-containing fibers below the site of injury was found in all animals treated with grafting and FGF1 in the lower thoracic cord. These fibers were detected more than $3 \mathrm{~mm}$ caudal to the injury indicating axonal elongation beyond the site of injury in the following one or two cord segments (Fig. 6i). BDA fibers were found in both central and peripheral parts 
Bilateral MEP recovery success rate

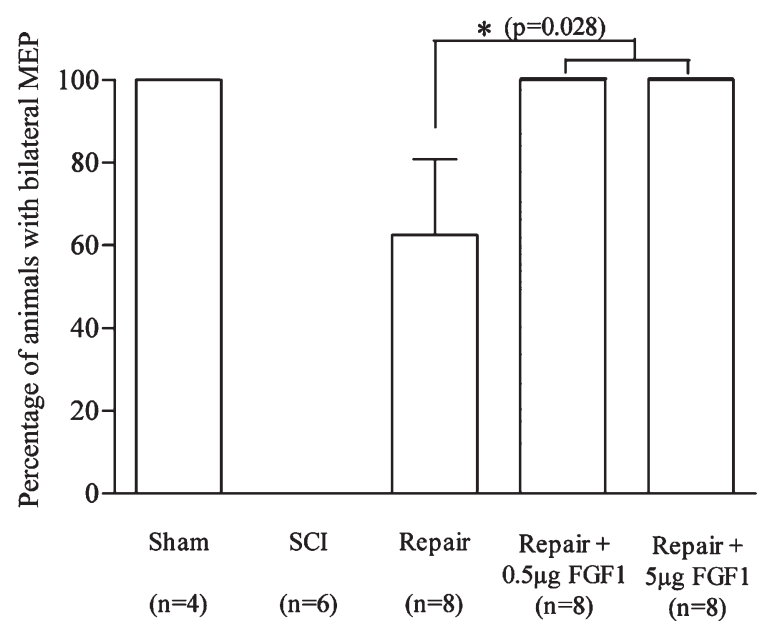

Fig. 5. Bilateral MEP recovery 20 weeks after surgery (mean \pm SEM), describing the fraction of animals with both left- and right-sided MEP response after contralateral cortex stimulation. All sham-operated animals showed full MEP responses after 20 weeks. In the repair groups treated with FGF1 $(5 \mu \mathrm{g}$ or $0.5 \mu \mathrm{g})$ and nerve grafts, all $(100 \%)$ animals showed positive bilateral MEPs, which was significantly better ( $p=0.028$, Fisher's exact test) than in the repair group not treated with FGF1, where five out of eight $(62.5 \%)$ animals showed positive bilateral MEPs, which was significantly better than the SCI $(0 \%)$ control group $(p=0.031$, Fisher's exact test).

of the caudal cord, which is line with the findings of Tsai and co-workers, who repaired the spinal cord with obliquely projected nerve graft or spinal cord fusion and found BDA traced fibers in both gray and white matter. No BDA was detected in the spinal cord below the lesion in control animals (Fig. 6j).

\subsection{Immunocytochemistry}

All nerve grafts within the injury zone contained numerous neurofilament (NF)-positive axons at 20 weeks post injury, most likely representing regenerating axons, either ascending or descending (Fig. 6b). Caudal to the graft area numerous NF labeled axons appeared in the grey matter (Fig. 6c). These axons had no clear organization and were projected in all directions in a turning and winding manner, resembling sprouting axons. We suggest that these fibers represent regenerating axons in line with the tracing results above, even though neurofilament staining cannot separate between sprouting ascending sensory axons from the transected caudal surface and descending fibers entering the caudal spinal cord. Glial fibrillary acidic protein (GFAP) immunoreactivity (IR) showed scattered astrocytes in all groups in the normal spinal cord or more than $1 \mathrm{~mm}$ away from any repair area in the various treatment groups (Fig. 7). Within the degraded repair area in treatment groups only few astrocytes were found. At the cranial and caudal resection borders in spinal cord resected animals, there was an increased GFAP-IR at study termination regardless of repair strategy, with no obvious difference among the groups.

\subsection{Semithin sections}

Numerous axons were found in all grafts 20 weeks postoperatively (Fig. 6d-f). The axons were of varying diameter, with different degree of myelination and were to a large extent found to be arranged in fascicles (Fig 6d-f).

\section{Discussion}

Although several promising regeneration strategies have been presented in recent years (Bunge, 2008; Cadotte and Fehlings, 2010; Cote, et al., 2011; Kwon, et al., 2010; Schwab, et al., 2006) spinal cord injury (SCI) remains a major challenge. One is to establish regeneration across a spinal cord gap by letting the long tracts in the spinal cord climb in peripheral nerve grafts (PNG) and enter permissive gray substance on the other side of the lesion (Cheng, et al., 1996; Cheng et al., 2004; Houle et al., 2006; Lee, et al., 2002; Lee, et al., 2004; Tsai, et al., 2005). To facilitate the microsurgical positioning of the PNGs, we previously developed a graft holder to facilitate reproducible placement of PNGs (Nordblom, et al., 2009). If there is a potential clinical application using PNGs, literature suggests that fibroblast growth factor 1 (FGF1) is needed for functional recovery (Cheng, et al., 1996; Lee, et al., 2002; Lee, et al., 2004; Lee et al., 2010; Tsai, et al., 2005). Therefore, the current investigation evaluated spinal cord repair with PNGs in a newly developed graft holder made of biodegradable calcium sulphate with or without slow release of FGF1.

A complete injury of the spinal cord was made by resection of a thoracic segment (T11) in adult rats followed by the implantation of a device carrying 12 autologous PNGs with or without FGF1. Postmortem findings demonstrated that the device was well 

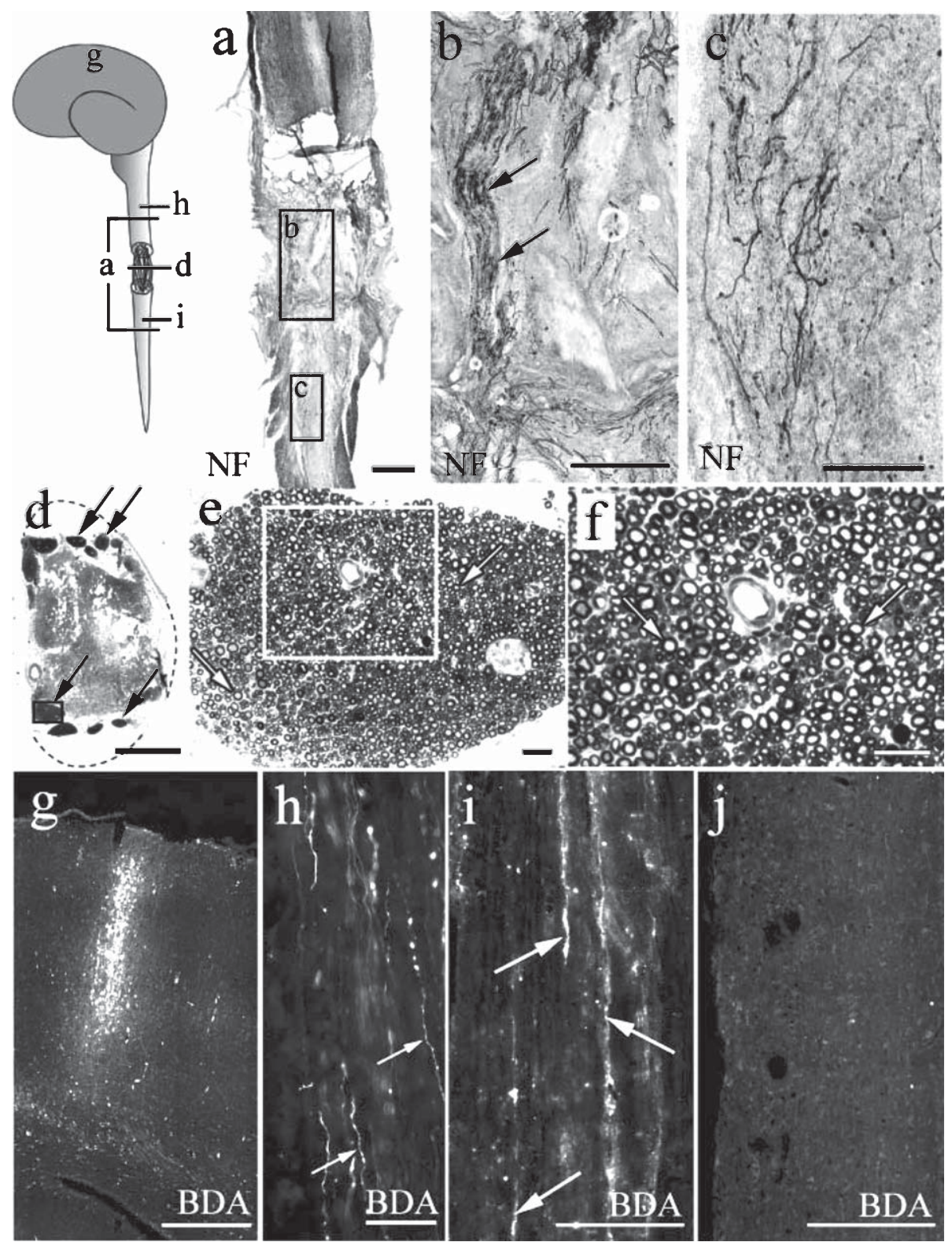

Fig. 6. Schematic illustration indicating the region ( $\mathrm{a}, \mathrm{d}, \mathrm{g}, \mathrm{h}, \mathrm{i})$ for micrographs showing neurofilament (NF, a-c) immunoreactivity (IR), semithin sections (d-f) and BDA (g-j) at 20 weeks after spinal cord repair with high dose FGF1. a: Low magnification showing the cranial cord, repair area (b) and the caudal (c) spinal cord. b: High magnification of a peripheral nerve graft in the degraded device, containing numerous NF-positive axons (arrows). Further down, numerous winding and turning NF-positive fibers were found $3 \mathrm{~mm}$ into the caudal spinal cord (c), most likely representing sprouting axons of unknown origin. d: Transverse section of the right side of the degraded device showing several transplanted nerve grafts at the outer border of the device area (arrows). e: High magnification of one nerve graft (black left corner box in d) revealed numerous well myelinated axons (arrows), the square is found enlarged in $\mathrm{f}$ (arrows). The presence of BDA at various locations (see schematic illustration at top left corner) following injection in the right motor cortex showed clearly labeled pyramidal cells $(\mathrm{g})$ and labeled axons in the dorsal corticospinal tract in the proximal spinal cord (h, arrows) at 10 weeks after surgery. Caudal to the SCI, there were several axons positive for BDA, up to $3 \mathrm{~mm}$ into the spinal cord (i, arrows) in animals subjected to spinal cord injury and repair, but not after SCI only (f). Bars = $1 \mathrm{~mm}(\mathrm{a}, \mathrm{d}), 50 \mu \mathrm{m}(\mathrm{b}, \mathrm{e}, \mathrm{f}), 30 \mu \mathrm{m}$ (c), $0.5 \mathrm{~mm}(\mathrm{~g}), 0.2 \mathrm{~mm}(\mathrm{~h}, \mathrm{i}, \mathrm{j})$. 


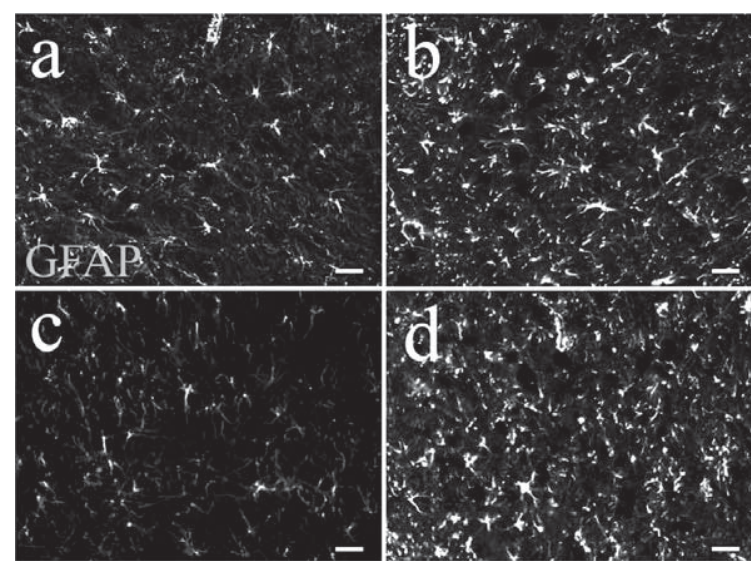

Fig. 7. Glial fibrillary acidic protein (GFAP) immunoreactivity (IR) in the caudal spinal cord at 20 weeks after sham operation (a), repair with high dose FGF1 (b-c) or repair without FGF1 (d). In sham operated animals only scattered astrocytes were found in the cord, whereas at the interface between the degraded device and caudal cord, there was an increased GFAP-IR (b), not noticeably different from animals repaired without FGF1 (d). One mm into the caudal cord (c), the GFAP-IR was similar to the cords of the sham group. $\operatorname{Bars}(\mathrm{a}-\mathrm{d})=50 \mu \mathrm{m}$

degraded and integrated in the spinal cord at 20 weeks after repair and that no apparent reaction toward the material was observed. Further, FGF1 augmented the electrophysiological response if the cord was reconnected with PNGs. According to previous literature, the effect of FGF1 may be attributed to neuroprotection, improved regeneration or local modulation of the spinal cord injury milieu (Giacobini et al., 1991; Guest et al., 1997; Kuo et al., 2011; Lee, et al., 2008; Lee et al., 2011; Tsai et al., 2008).

We also report that the electrophysiological connection between the motor cortex and hind limb muscles was paralleled by traced corticospinal tract (CST) axons into the caudal spinal cord. This demonstrates the principle that CST axons may enter and exit PNGs in the spinal cord repair in the presence of FGF1, and serves as an additional piece of evidence that the motor cortex is involved in the recovery after spinal cord repair with PNGs. Putative effect of FGF1 purely on CST regeneration was not assessed in the current study. SCI alone showed no CST axons in the caudal cord. The positive MEPs were generated from the same place as the BDA injections were made in the motor cortex. Even though it is likely that the CST fibers conduct some of the signals that give rise to the positive MEPs in this study, other reasonable MEP generating pathways are possible. Such pathways could be polysynaptic circuits through brainstem derived signals or propriospinal axons in the spinal cord traversing the injury zone in the PNG, as well as other long tract terminations in neuron pools in the caudal spinal cord before connecting to the secondary motor neurons targeting hind limb muscles. If CST fibers would terminate directly onto alpha motor neurons to the hamstring muscles (in the rat innervated mainly by the L5 segment) regeneration distance would have to be about $20 \mathrm{~mm}$ from the cranial injury zone into the caudal cord (post mortem examination of distance to L5 segment, data not shown).

The MEP response in the hind limb muscles were recorded after increasing stimulation until response was registered or terminated at $30 \mathrm{~mA}$. All positive MEPs disappeared after cord re-transection at the T8 level. During MEP sessions, several stimulations were made with the bipolar electrode on the cortex giving a variation of amplitudes, which is dependent on the amount of depolarized neurons in the cortex (bipolar electrode placement) as well as the muscle cell depolarization around a specific volume around the muscle needle, i.e. needle position dependent. Therefore the MEPs could only be reported as cortex-hind limb contact or not (one or zero response), which is in line with literature on MEPs describing large variations of amplitudes seen in the same subject at different time points depending on stimulation and registration coordinates (Wasserman, 2008). Thus, it may be misleading to compare MEP amplitudes in the current experimental setting.

There is much data to confirm that PNGs is a successful substrate for regeneration stimulation of the peripheral or central nervous system (Alilain, et al., 2011; Cheng, et al., 1996; Cote, et al., 2011; Richardson et al., 1980). Therefore the current study only evaluated spinal cord bridging repair strategies based on PNGs in a biodegradable graft holder with two different concentrations of FGF1 or without. Thus, the beneficial effect of FGF1 addition to the graft holder could only be attributed to PNG/FGF1 in combination and not separate the individual contribution from PNG or FGF1 alone.

All grafted animals presented with BBB scores between 0 and 4 from the 6 th postoperative week. This was significantly better than injured animals without repair suggesting that a limited functional regeneration had occurred. The reported BBB scores in treated animals are somewhat lower than BBB scores reported by Tsai and coworkers (BBB of 4-5) (Tsai, et al., 
2005) or Lee et al. (BBB around 7) (Lee, et al., 2002; Lee, et al., 2004) after spinal cord injury repair with PNGs and FGF1. However, it is difficult to grade repair strategies comparing treatments groups from separate papers, due to possible differences in e.g. origin of rats, care or rehabilitation milieu (Garrison et al., 2011; Lee, et al., 2010). Functional recovery after CNS injury is also reported to differ depending on rat strain. (Reid et al., 2010). The current groups of rats suffered from severe contractures and fixed joints, and a possibly emerging BBB increase could have been disguised. They were not subjected to physiotherapy of the joints. Efficiency grading among the various reports may include several pitfalls and is currently omitted.

The current paper suggests that the addition of FGF1 in the biodegradable graft holder improves the electrophysiological response, but is not designed to evaluate the effectiveness of PNGs, since nerve grafts were used in all treatment groups. In the future, there will be interest in separating the impact of the individual components FGF1 and PNGs in spinal cord repair, which was beyond the scope of the present study.

In conclusion, we report a tolerable method to bridge a spinal cord gap with PNGs with high microsurgical precision in a biodegradable device. If FGF1 was added in the calcium sulphate, MEP appearance was more robust than without FGF1. The method was paralleled with a functional improvement and evidence of CST axons traversing the repair area and into the caudal spinal cord. If the method enters a course toward a translational approach, irreversibly and completely devitalized spinal cord tissue would have to be removed, which demands an unambiguous diagnosis of complete spinal cord injury. (Steeves et al., 2010; Zariffa et al., 2010).

\section{Acknowledgments}

We would like to thank Britt Meijer, Håkan Eriksson and Björn Hellberg for excellent laboratory assistance, Brita Robertson for good advices on tracing techniques and Jeremy Young for MRI assistance. This work was supported by the Karolinska Institute, Swedish Medical Research Council, Torsten and Ragnar Söderberg's foundation, Socialstyrelsen, Magnus Bergwall's foundation, The Swedish Association of Persons with Neurological Disabilities, VINNOVA and BioArctic Neuroscience AB.

\section{Author disclosure statement}

J. Sjödahl works for BioArctic AB, which has a financial interest in spinal cord repair. No other author of this article has any financial conflict of interest in this publication.

\section{References}

Aberg, J., Eriksson, O., Spens, E., Nordblom, J., Mattsson, P., Sjodahl, J., Svensson, M. \& Engqvist, H. (2010). Calcium sulfate spinal cord scaffold: A study on degradation and fibroblast growth factor 1 loading and release. J Biomater Appl.

Alilain, W.J., Horn, K.P., Hu, H., Dick, T.E. \& Silver, J. (2011). Functional regeneration of respiratory pathways after spinal cord injury. Nature, 475(7355), 196-200.

Basso, D.M., Beattie, M.S. \& Bresnahan, J.C. (1995). A sensitive and reliable locomotor rating scale for open field testing in rats. J Neurotrauma, 12(1), 1-21.

Bunge, M.B. (2008). Novel combination strategies to repair the injured mammalian spinal cord. J Spinal Cord Med, 31(3), 262-269.

Cadotte, D.W. \& Fehlings, M.G. (2011). Spinal Cord Injury: A Systematic Review of Current Treatment Options. Clin Orthop Relat Res, 469(3), 732-741.

Chen, M.S., Huber, A.B., van der Haar, M.E., Frank, M., Schnell, L., Spillmann, A.A., Christ, F. \& Schwab, M.E. (2000). Nogo-A is a myelin-associated neurite outgrowth inhibitor and an antigen for monoclonal antibody IN-1. Nature, 403(6768), 434-439.

Cheng, H., Cao, Y. \& Olson, L. (1996). Spinal cord repair in adult paraplegic rats: Partial restoration of hind limb function. Science, 273(5274), 510-513.

Cheng, H., Liao, K.K., Liao, S.F., Chuang, T.Y. \& Shih, Y.H. (2004). Spinal cord repair with acidic fibroblast growth factor as a treatment for a patient with chronic paraplegia. Spine, 29(14), E284-E288.

Cote, M.P., Amin, A.A., Tom, V.J. \& Houle, J.D. (2011). Peripheral nerve grafts support regeneration after spinal cord injury. Neurotherapeutics, 8(2), 294-303

Fehlings, M.G., Theodore, N., Harrop, J., Maurais, G., Kuntz, C., Shaffrey, C.I., Kwon, B.K., Chapman, J., Yee, A., Tighe, A. \& McKerracher, L. (2011). A phase I/IIa clinical trial of a recombinant Rho protein antagonist in acute spinal cord injury. J Neurotrauma, 28(5), 787-796.

Fitch, M.T. \& Silver, J. (2008). CNS injury, glial scars, and inflammation: Inhibitory extracellular matrices and regeneration failure. Exp Neurol, 209(2), 294-301.

Garrison, M.K., Yates, C.C., Reese, N.B., Skinner, R.D. \& GarciaRill, E. (2011). Wind-up of stretch reflexes as a measure of spasticity in chronic spinalized rats: The effects of passive exercise and modafinil. Exp Neurol, 227(1), 104-109.

Giacobini, M.M., Hoffer, B.J., Zerbe, G. \& Olson, L. (1991). Acidic and basic fibroblast growth factors augment growth of fetal brain tissue grafts. Exp Brain Res, 86(1), 73-81. 
Guest, J.D., Hesse, D., Schnell, L., Schwab, M.E., Bunge, M.B. \& Bunge, R.P. (1997). Influence of IN-1 antibody and acidic FGFfibrin glue on the response of injured corticospinal tract axons to human Schwann cell grafts. J Neurosci Res, 50(5), 888-905.

Harkema, S., Gerasimenko, Y., Hodes, J., Burdick, J., Angeli, C., Chen, Y., Ferreira, C., Willhite, A., Rejc, E., Grossman, R.G. \& Edgerton, V.R. (2011). Effect of epidural stimulation of the lumbosacral spinal cord on voluntary movement, standing, and assisted stepping after motor complete paraplegia: A case study. Lancet, 377(9781), 1938-1947.

Hennig, J., Nauerth, A. \& Friedburg, H. (1986). RARE imaging: A fast imaging method for clinical MR. Magn Reson Med, 3(6), 823-833.

Houle, J.D., Tom, V.J., Mayes, D., Wagoner, G., Phillips, N. \& Silver, J. (2006). Combining an autologous peripheral nervous system "bridge" and matrix modification by chondroitinase allows robust, functional regeneration beyond a hemisection lesion of the adult rat spinal cord. $J$ Neurosci, 26(28), 7405-7415.

Kuo, H.S., Tsai, M.J., Huang, M.C., Chiu, C.W., Tsai, C.Y., Lee, M.J., Huang, W.C., Lin, Y.L., Kuo, W.C. \& Cheng, H. (2011). Acid fibroblast growth factor and peripheral nerve grafts regulate Th2 cytokine expression, macrophage activation, polyamine synthesis, and neurotrophin expression in transected rat spinal cords. $J$ Neurosci, 31(11), 4137-4147.

Kwon, B.K., Sekhon, L.H. \& Fehlings, M.G. (2010). Emerging repair. regeneration, and translational research advances for spinal cord injury. Spine (Phila Pa 1976), 35(21 Suppl), S263S270.

Lee, M.J., Chen, C.J., Cheng, C.H., Huang, W.C., Kuo, H.S., Wu, J.C., Tsai, M.J., Huang, M.C., Chang, W.C. \& Cheng, H. (2008). Combined treatment using peripheral nerve graft and FGF-1: Changes to the glial environment and differential macrophage reaction in a complete transected spinal cord. Neurosci Lett, 433(3), 163-169.

Lee, M.J., Chen, C.J., Huang, W.C., Huang, M.C., Chang, W.C., Kuo, H.S., Tsai, M.J., Lin, Y.L. \& Cheng, H. (2011). Regulation of chondroitin sulphate proteoglycan and reactive gliosis after spinal cord transection: Effects of peripheral nerve graft and fibroblast growth factor 1. Neuropathol Appl Neurobiol, 37(6), 585-599.

Lee, Y.S., Hsiao, I. \& Lin, V.W. (2002). Peripheral nerve grafts and aFGF restore partial hindlimb function in adult paraplegic rats. J Neurotrauma, 19(10), 1203-1216.

Lee, Y.S., Lin, C.Y., Robertson, R.T., Hsiao, I. \& Lin, V.W. (2004). Motor recovery and anatomical evidence of axonal regrowth in spinal cord-repaired adult rats. J Neuropathol Exp Neurol, 63(3), 233-245

Lee, Y.S., Zdunowski, S., Edgerton, V.R., Roy, R.R., Zhong, H., Hsiao, I. \& Lin, V.W. (2010). Improvement of gait patterns in step-trained, complete spinal cord-transected rats treated with a peripheral nerve graft and acidic fibroblast growth factor. Exp Neurol, 224(2), 429-437.

Nordblom, J., Persson, J.K., Svensson, M. \& Mattsson, P. (2009). Peripheral nerve grafts in a spinal cord prosthesis result in regeneration and motor evoked potentials following spinal cord resection. Restor Neurol Neurosci, 27(4), 285-295.

Reid, W.M., Rolfe, A., Register, D., Levasseur, J.E., Churn, S.B. \& Sun, D. (2010). Strain-related differences after experimental traumatic brain injury in rats. J Neurotrauma, 27(7), 1243-1253.

Richardson, P.M., McGuinness, U.M. \& Aguayo, A.J. (1980). Axons from CNS neurons regenerate into PNS grafts. Nature, 284(5753), 264-265.

Schwab, J.M., Brechtel, K., Mueller, C.A., Failli, V., Kaps, H.P., Tuli, S.K. \& Schluesener, H.J. (2006). Experimental strategies to promote spinal cord regeneration-an integrative perspective. Prog Neurobiol, 78(2), 91-116.

Schwab, M.E. (2004). Nogo and axon regeneration. Curr Opin Neurobiol, 14(1), 118-124.

Steeves, J.D., Kramer, J.K., Fawcett, J.W., Cragg, J., Lammertse, D.P., Blight, A.R., Marino, R.J., Ditunno, J.F. Jr, Coleman, W.P., Geisler, F.H., Guest, J., Jones, L., Burns, S., Schubert, M., van Hedel, H.J. \& Curt, A. (2011). Extent of spontaneous motor recovery after traumatic cervical sensorimotor complete spinal cord injury. Spinal Cord, 49(2), 257-265.

Tsai, E.C., Krassioukov, A.V. \& Tator, C.H. (2005). Corticospinal regeneration into lumbar grey matter correlates with locomotor recovery after complete spinal cord transection and repair with peripheral nerve grafts, fibroblast growth factor 1, fibrin glue, and spinal fusion. J Neuropathol Exp Neurol, 64(3), 230-244.

Tsai, M.C., Shen, L.F., Kuo, H.S., Cheng, H. \& Chak, K.F. (2008) Involvement of acidic fibroblast growth factor in spinal cord injury repair processes revealed by a proteomics approach. Mol Cell Proteomics, 7(9), 1668-1687.

Wassermann, E.M., Epstein, C.M., Ziemann, U., Walsh, V., Paus, T. \& Lisanby, S. (2008). The Oxford Handbook of Transcranial Stimulation (1 ed.). New York: Oxford University Press.

Wu, J.C., Huang, W.C., Chen, Y.C., Tu, T.H., Tsai, Y.A., Huang, S.F., Huang, H.C. \& Cheng, H. (2011). Acidic fibroblast growth factor for repair of human spinal cord injury: A clinical trial. $J$ Neurosurg Spine, 15(3), 216-227.

Zariffa, J., Kramer, J.L., Fawcett, J.W., Lammertse, D.P., Blight, A.R., Guest, J., Jones, L., Burns, S., Schubert, M., Bolliger, M., Curt, A. \& Steeves, J.D. (2011). Characterization of neurological recovery following traumatic sensorimotor complete thoracic spinal cord injury. Spinal Cord, 49(3), 463-471. 